\title{
Skeletal Muscle Weakness Is Associated With Both Early and Late Mortality After Acute Respiratory Distress Syndrome*
}

\author{
Zudin Puthucheary, MRCP, PhD \\ Division of Critical Care \\ Institute of Sports and Exercise Health \\ University College London Hospitals \\ London, UK
}

\author{
Hallie Prescott, MD, MSC \\ Division of Pulmonary and Critical Care Medicine \\ University of Michigan Health System; and \\ HSR\&D Center for Clinical Management Research \\ VA Ann Arbor Healthcare System \\ Ann Arbor, MI
}

r n 2003, Herridge et al (1) first described poor functional outcomes in working-age, 1-year survivors of acute respiratory distress syndrome (ARDS). In the ensuring decade, countless studies have confirmed that poor long-term outcomes are the rule, not the exception, after ARDS. Survivorship from critical illness has been heralded as the defining challenge of critical care in the 21 st century (2). Recently, helping patients to thrive after critical illness was deemed the "third revolution" of critical care (3).

As the field of critical care medicine increasingly focuses on long-term outcomes, it is important to recognize that long-term outcomes are the result of two distinct components: early and late events (Fig. 1). For example, 5-year mortality is the result of early mortality (e.g., in-hospital death) and late mortality (e.g., death after hospital discharge but within $5 \mathrm{yr}$ of hospitalization). If early mortality is sufficiently high enough, then longterm mortality will be high even if the critical illness does not increase risk for late death among those who survive to hospital discharge. When considering long-term mortality from critical illness, it is important to differentiate between early and late deaths because they are driven by different factors (4).

While early mortality from ARDS has been steadily declining with time (5), late mortality-death in the years after ARDS hospitalization-remains high (6). Drivers of this late mortality are not well understood. As would be expected, age, preexisting chronic medical conditions, and prior nursing home use

\footnotetext{
*See also p. 446.

Key Words: acute respiratory distress syndrome; intensive care unit acquired weakness; muscle wasting

Dr. Prescott's institution received funding from the National Institutes of Health (NIH) and the American Thoracic Society Foundation. She received support for article research from the NIH. Dr. Puthucheary disclosed that he does not have any potential conflicts of interest.

Copyright (C) 2017 by the Society of Critical Care Medicine and Wolters Kluwer Health, Inc. All Rights Reserved.
}

DOI: 10.1097/CCM.0000000000002243 predict increased risk for late mortality (6). As yet, though, we have not elucidated if or how ARDS itself increases risk for late mortality relative to age and comorbidity-matched controls.

In studies with careful matching and control for confounding, pneumonia (7), sepsis (8), and all-cause critical illness (9) have each been found to increase survivor's risk for late mortality, independent of age, and baseline health status. So, we can reasonably hypothesize that surviving ARDS also increases one's risk for late mortality. But, the mechanisms by which any of these acute diseases increases late mortality remain poorly understood. Impaired immunity, weakness, accelerated cardiovascular disease, or heightened risk for malignancy may all play a role.

In this issue of Critical Care Medicine, Dinglas et al (10) examined the relationship of skeletal muscle weakness and late mortality in ARDS survivors - in effect, tying together outcomes which are often considered in isolation: functional disability and late mortality. Skeletal muscle wasting occurs rapidly in critical illness, is a major contributor to functional disability (11), and predicts early mortality (12). But, the impact of muscle weakness on late mortality in ARDS survivors has not been described previously.

One hundred fifty-six ARDS survivors were followed to death or for 5 years following hospital discharge. Muscle strength was measured at hospital discharge and again 3, 6, 12, 24, 36, and 48 months postdischarge using the Medical Research Council (MRC) sum score, a 0 to 60 strength scale where a score less than 48 is considered to reflect pathologic weakness. The MRC is recommended by several critical care professional societies to measure muscle strength but is a subjective test (13). To combat the known subjectivity of MRC, this study included rigorous quality control; strength assessors underwent initial training and periodic quality review for the duration of the study to ensure high interrater reliability of their assessments.

The authors examined the association of weakness at hospital discharge and changes in weakness over time (persistent, resolved, and new) with 5-year mortality. An initial multivariable model was used to inform a parsimonious set of covariates (age, comorbidity, and mean daily Sequential Organ Failure Assessment score) that were included in the final Cox proportional hazard model. Each of these covariates been previously described to affect either skeletal muscle loss/function (14) or mortality (15).

At hospital discharge, $38 \%$ of the ARDS survivors in this study had muscle weakness, and one third of the cohort died during the 5-year follow-up period. Muscle weakness at hospital discharge was independently associated with 5-year mortality. The association persisted whether weakness was considered as a binary predictor (being weak at hospital discharge predicted 5 -yr mortality) or as a continuous predictor (each additional one point loss of strength predicted higher 5-yr mortality). 


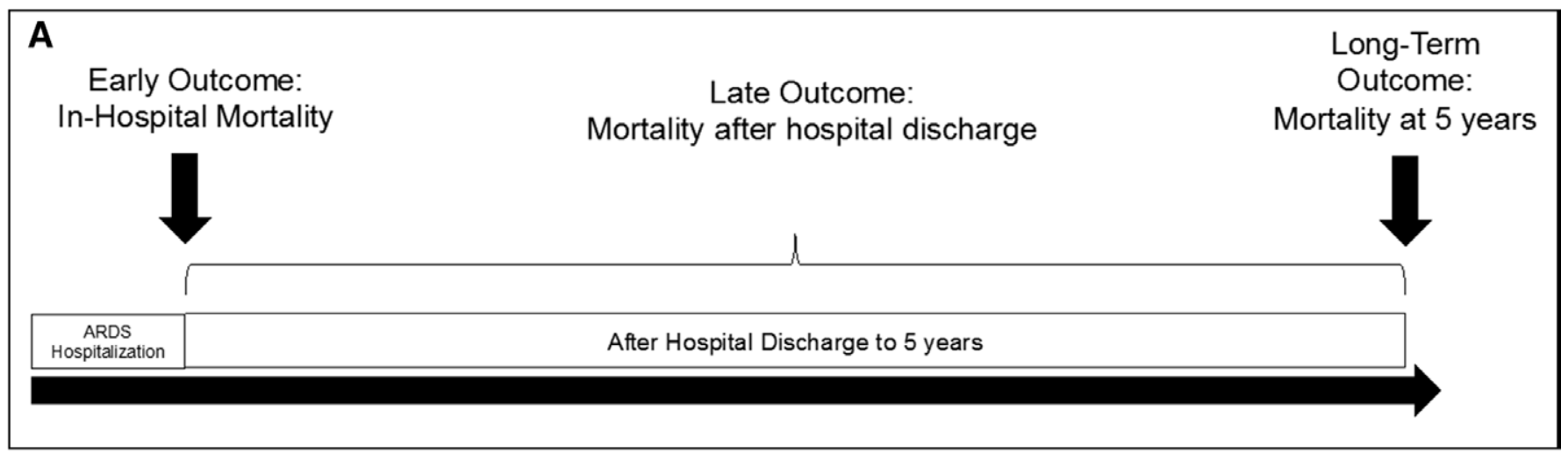

B

\section{Scenario 1: Increased Long-term Mortality, and increased Late Mortality}

\section{Scenario 2: Increased Long-term Mortality, but no increase in late mortality}
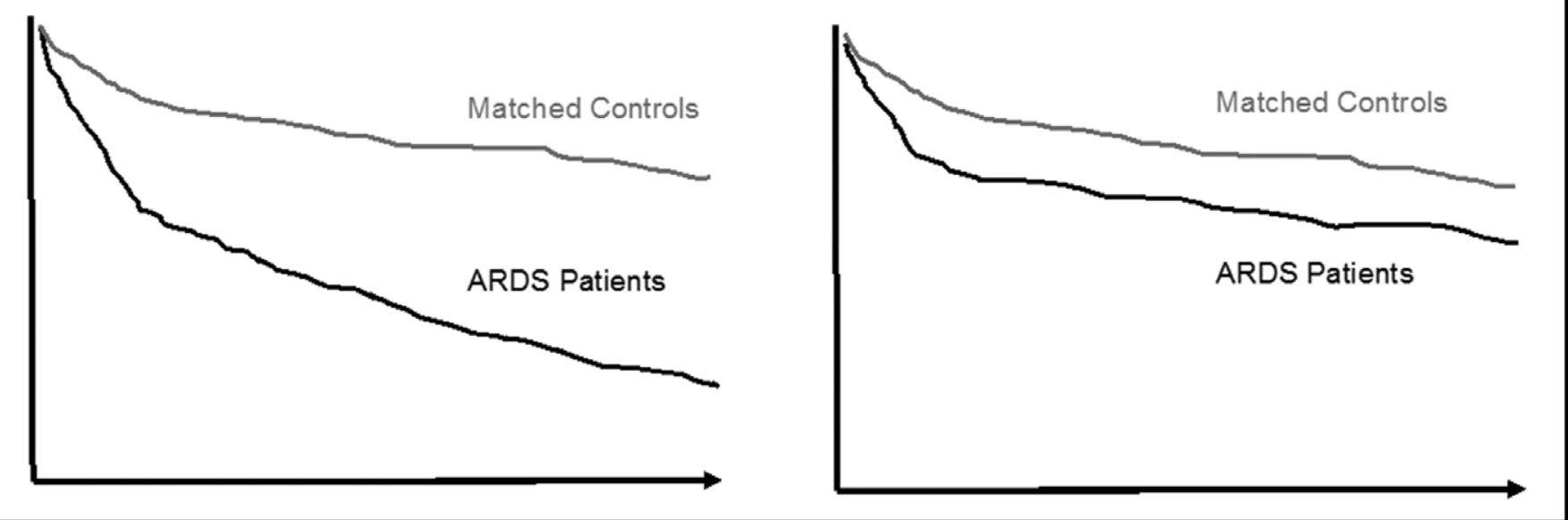

Figure 1. Long-term versus late mortality after critical illness. A, Components of long-term mortality. B, Hypothetical Kaplan-Meier survival curve for patients with acute respiratory distress syndrome (ARDS) and matched controls without ARDS. Long-term mortality after critical illness consists of early and late deaths. In this study, early deaths were considered as in-hospital deaths, whereas late deaths were deaths in the $5 \mathrm{yr}$ following survival from ARDS hospitalization. B shows that ARDS patients may have increased long-term mortality with (scenario 1) or without (scenario 2) increased late mortality.

Persistent weakness (maintaining MRC < 48) over time was associated with worse 5-year survival, compared with maintaining a normal MRC. But curiously, resolving weakness was no better than persistent weakness, suggesting that weakness at hospital discharge is an ominous sign, even if a patient is subsequently able to rehabilitate back to normal strength. In future work, it would be interesting to see the relationship of muscle weakness trajectories to late mortality stratified by physical function at hospital discharge (16) or inflammatory load during acute illness (17). Progressing from normal strength to weakness during the 5-year follow-up period was not associated with worse 5-year survival, but this trajectory had insufficient power to draw strong conclusions.

These data attractively suggest that muscle wasting is a unifying contributor to late mortality and functional disability. An alternative view may be that muscle weakness and weakness trajectories are epiphenomena of the more holistic concept of frailty (18), and that frailty drives late mortality. Yet, it is biologically plausible that muscle weakness itself increases late mortality. Muscle weakness may cause swallowing dysfunction
(19), decreased nutritional intake (19), increased aspiration (19), and decreased physical activity (20), which in turn may increase the risk for cardiovascular events and malignancy. Individually or in combination, these factors may impair muscle protein synthesis, leading to a vicious cycle of maintained cachexia-which is strongly associated with mortality (21).

As in-hospital mortality from ARDS continues to fall, we are increasingly challenged to improve the survivor experience. This study argues that skeletal muscle weakness is a key determinant of not only functional disability but also of late mortality. Even those patients whose weakness improved over time experienced increased rates of 5-year mortality, underscoring the importance of preventing muscle loss during the acute phase of critical illness.

\section{REFERENCES}

1. Herridge MS, Cheung AM, Tansey CM, et al; Canadian Critical Care Trials Group: One-year outcomes in survivors of the acute respiratory distress syndrome. N Engl J Med 2003; 348:683-693

2. Iwashyna TJ: Survivorship will be the defining challenge of critical care in the $21^{\text {st }}$ century. Ann Intern Med 2010; 153:204-205 
3. Iwashyna TJ, Speelmon EC: Advancing a third revolution in critical care. Am J Respir Crit Care Med 2016; 194:782-783

4. Garland A, Olafson K, Ramsey CD, et al: Distinct determinants of long-term and short-term survival in critical illness. Intensive Care Med 2014; 40:1097-1105

5. Zambon M, Vincent JL: Mortality rates for patients with acute lung injury/ARDS have decreased over time. Chest 2008; 133:1120-1127

6. Wang CY, Calfee CS, Paul DW, et al: One-year mortality and predictors of death among hospital survivors of acute respiratory distress syndrome. Intensive Care Med 2014; 40:388-396

7. Kaplan V, Clermont G, Griffin MF, et al: Pneumonia: Still the old man's friend? Arch Intern Med 2003; 163:317-323

8. Prescott HC, Osterholzer JJ, Langa KM, et al: Late mortality after sepsis: Propensity matched cohort study. BMJ 2016; 353:i2375

9. Lone NI, Gillies MA, Haddow C, et al: Five-year mortality and hospital costs associated with surviving intensive care. Am J Respir Crit Care Med 2016; 194:198-208

10. Dinglas VD, Aronson Friedman L, Colantuoni E, et al: Muscle Weakness and 5-Year Survival in Acute Respiratory Distress Syndrome Survivors. Crit Care Med 2017; 45:446-453

11. Parry SM, El-Ansary D, Cartwright MS, et al: Ultrasonography in the intensive care setting can be used to detect changes in the quality and quantity of muscle and is related to muscle strength and function. J Crit Care 2015; 30:1151.e9-1151.14

12. Hermans G, Van Mechelen $H$, Clerckx B, et al: Acute outcomes and 1-year mortality of intensive care unit-acquired weakness. A cohort study and propensity-matched analysis. Am J Respir Crit Care Med $2014 ; 190: 410-420$
13. Hough CL, Lieu BK, Caldwell ES: Manual muscle strength testing of critically ill patients: Feasibility and interobserver agreement. Crit Care $2011 ; 15: R 43$

14. Puthucheary ZA, Rawal J, McPhail M, et al: Acute skeletal muscle wasting in critical illness. JAMA 2013; 310:1591-1600

15. Lone NI, Walsh TS: Impact of intensive care unit organ failures on mortality during the five years after a critical illness. Am J Respir Crit Care Med 2012; 186:640-647

16. Puthucheary ZA, Denehy L: Exercise interventions in critical illness survivors: Understanding inclusion and stratification criteria. Am J Respir Crit Care Med 2015; 191:1464-1467

17. Griffith DM, Lewis S, Rossi AG, et al; RECOVER Investigators: Systemic inflammation after critical illness: Relationship with physical recovery and exploration of potential mechanisms. Thorax 2016; 71:820-829

18. Bagshaw SM, Stelfox HT, McDermid RC, et al: Association between frailty and short- and long-term outcomes among critically ill patients: A multicentre prospective cohort study. CMAJ 2014; 186:E95-102

19. Mirzakhani H, Williams JN, Mello J, et al: Muscle weakness predicts pharyngeal dysfunction and symptomatic aspiration in long-term ventilated patients. Anesthesiology 2013; 119:389-397

20. McNelly AS, Rawal J, Shrikrishna D, et al: An exploratory study of long-term outcome measures in critical illness survivors: Construct validity of physical activity, frailty, and health-related quality of life measures. Crit Care Med 2016; 44:e362-e369

21. Kalantar-Zadeh K, Rhee C, Sim JJ, et al: Why cachexia kills: Examining the causality of poor outcomes in wasting conditions. J Cachexia Sarcopenia Muscle 2013; 4:89-94 